\title{
A Generic Middleware Model for Smart Home
}

\author{
Madhusudanan J. \\ Research Scholar, Pondicherry University, Puducherry, India \\ Email: contactmadhu@gmail.com \\ Hariharan S. \\ PG Scholar, Sri Manakula Vinayagar Engineering College, Puducherry, India \\ Email: hari1691@gmail.com \\ Manian A. Selvan \\ UG Scholar, Sri Manakula Vinayagar Engineering College, Puducherry, India \\ Email: manianselvan@gmail.com \\ Dr. V. Prasanna Venkatesan \\ Associate Professor, Pondicherry University, Puducherry, India \\ Email: prasanna_v@yahoo.com
}

\begin{abstract}
A Smart Home is an emerging technology, where the electronic devices are controlled automatically based on the occupants activities. The pervasive computing plays a vital role in the smart home environment, which provides the computer-based service to human beings anywhere and anytime. However, when discussing smart home of the future, related studies have focused on providing middleware. The middleware acts as a interface between human beings and the smart devices. In this paper, we have proposed a generic middleware model for smart home that enables interaction between human being and devices and also between various devices based on the context identified in the environment.
\end{abstract}

Index Terms - Smart Home, Generic Middleware Model, Context.

\section{INTRODUCTION}

A "smart home" ${ }^{[1]}$ is defined as a residence or living environment equipped with the devices having the capability of computing and communication which predicts the activity of the occupant and responds to the needs of the occupants. The devices work to improve the comfort, convenience, security of the occupant through the technology management inside the home and connections to the world beyond.

The smart home ${ }^{[2]}$ equipped with the information $n$ technology and communication system also provides the occupant with high level of understanding and control which is not possible in the traditional "dump" homes. Generally, Smart Home includes devices with the capable of sensing the occupant activity and acts automatically to provide service needed by the occupant. The smart home provides interaction between the devices and the occupant and also among the various devices in the environment. There is the possibility of two way information flow between the occupants and the devices. The control system in the smart home allows the user to change or modify the operation that has to be carried by the devices according to his/her needs.

The smart home environment is only possible with the combination of various domains like pervasive computing, wireless sensor networks, etc. The pervasive computing vision ${ }^{[3-4]}$ is to provide computer based service to users anywhere (Ubiquitous). The services provided must be invisible to the users. The pervasive computing is also called as Ubiquitous computing or ambient intelligence or every ware. The wireless sensor also plays an important role in the smart home environment. The devices in the smart home environment are enabled with sensors and actuators to identify the needs of the occupants and to make response to them. For instance, the sensors can be used for various purposes like identifying climate, room temperature, lighting, etc. Based on the situation, response can be made automatically by the devices with the help of actuators. Mostly, Smart Home ${ }^{[5]}$ systems are used to monitor and serve the elder people ${ }^{[6]}$ or physically challenged occupants.

This paper is organized as follows: Section II presents a related work, Section III presents the Proposed Model of Generic Ubiquitous Middleware for smart home, Section IV represents Evaluation of proposed model using various smart home environments and Section V presents conclusion of the work

\section{RELATED WORKS}

The automation of the smart Home environment includes deploying various smart devices (i.e.) the devices with sensors and actuators and also providing service automatically to the need of the occupants. For performing such a thing needs the use of middleware for building smart home environment, where smart devices 
can be added and testing of scenario in smart home environment with the occupant's context. The various middleware like CASAS [7], PACE ${ }^{[8-9]}$, Atlas ${ }^{[10-11]}$, Hydra ${ }^{[12]}$, CDTOM ${ }^{[13]}$, etc. are proposed for the building of smart home environments.

Generally, in smart home actuation (i.e.) response to the service requested by the occupant is difficult. It requires middleware platform to support actuators.

Some platforms support actuators, but have constraints like scalability and fixed hardware configuration. Atlas is a kind of platform which supports both the sensors and actuators in the smart home environment which enables self integration and programmable pervasive or active spaces concepts with the capability to represent the connected devices as software services automatically. Atlas also provides physical nodes to connect heterogeneous devices.

Smart Home must be capable of changing its behavior dynamically based on the user's activities and the environment. Awareness about the environment and user's operation is required. To provide this, context aware task-oriented middleware (CDTOM) was designed. It provides service by abstracting the task from the routine activity with the intended goals. The CDTOM middleware provides various services like context storage, context data acquisition, context driven task reasoning, service discovery and task oriented mapping.

The CASAS is a kind of middleware or platform which provides a lightweight design for smart home that is easy to install and to provide the services of smart home capabilities without any customizations or training. CASAS is also referred as "Smart Home in a Box", which is designed like a smart home kit in a small form. It is also lightweight infrastructure, but it can be extendable with only minimal efforts. This kind of platform can be useful for building a smart home in a specific context, this is the problem identified in it.

For accommodating legacy components like transmitters and receivers in the handover system, the middleware called PACE was developed. In PACE, context and preferred repositories can be discovered dynamically with the help of mobile context aware components using various service discovery protocols. The privacy is provided in PACE middleware by allowing access control for context information's that are sensitive. The major problems in PACE middleware are scalability and fault tolerance.

\section{THE PROPOSED MODEL}

This chapter provides the detailed description of the Generic middleware model. The Generic ubiquitous middleware model allows the user to design the smart home environment of their requirement with the generic context. The middleware that are discussed in the previous chapter have the disadvantage of building a smart home environment only for a specific context. And also have drawbacks like scalability, fault tolerance, etc. The generic ubiquitous middleware provides a model to construct various kind of smart home environment of the users need.

The Generic Middleware ${ }^{[14]}$ model is constructed with four layers namely device, user, location and environment which is shown in the below figure 1.

A Generic Ubiquitous Middleware ${ }^{[15-16]}$ model for smart Home is constructed based on the two aspects namely context and device which is represented as the inner layer of the model as shown in the figure 1. Context is any information related to the entity's situation or scenario. The entity can be a person, place or objects that involves in the interaction of the users and the devices in the specified environments. The context can be classified into various kinds like User context, location context, Device context, Operation context, time context, etc based on the environment. For example, consider a smart home. The contexts involved in the smart home environment are User Context, location context, device context, environment context, operation context, time context, etc. Since model is generic, any kind contexts can be added or removed based on the users or occupants requirements.

The device aspect of the generic middleware model of Smart home environment deals with the infrastructure building of smart home with various devices like sensors, actuators in the specified location of the smart home environment to provide the services required by the users or occupants. The four layers of generic middleware model are explained in the detail as follows.

\section{A. User Layer}

The user layer of generic ubiquitous middleware model for smart home depicts the possible users that can occupy the generic smart home environment like owner, guest, friends, relatives, electrician, plumber, watchman, gardener, Housekeepers, neighbors, etc. The User layer in the generic middleware model for smart home provides the capability of adding any kind of users to the environment based on the context.

\section{B. Device Layer}

The device layer in the generic ubiquitous middleware model of smart home shows the devices or appliances that can be used in the home environment. Some of the devices that are available in smart home environment are light, fan, AC, refrigerator, washing machine, television, music player, micro-oven, computer, etc. The device will actuate according to the users context. The device layer in generic middleware model of smart home provides the proficiency of adding various devices to the smart home environment with users need.

\section{Location Layer}

The location layer in generic smart home middleware model represents the possible locations that can be available in the various smart home environments. The smart home can be built with various locations like hall, 
bedroom, staircase, wash room, bath room, garden, parking, kitchen, store room, balcony, dining hall, etc. It also provides the proficiency of adding any kind of location to the smart home environment which helps to provide generic smart home environments.

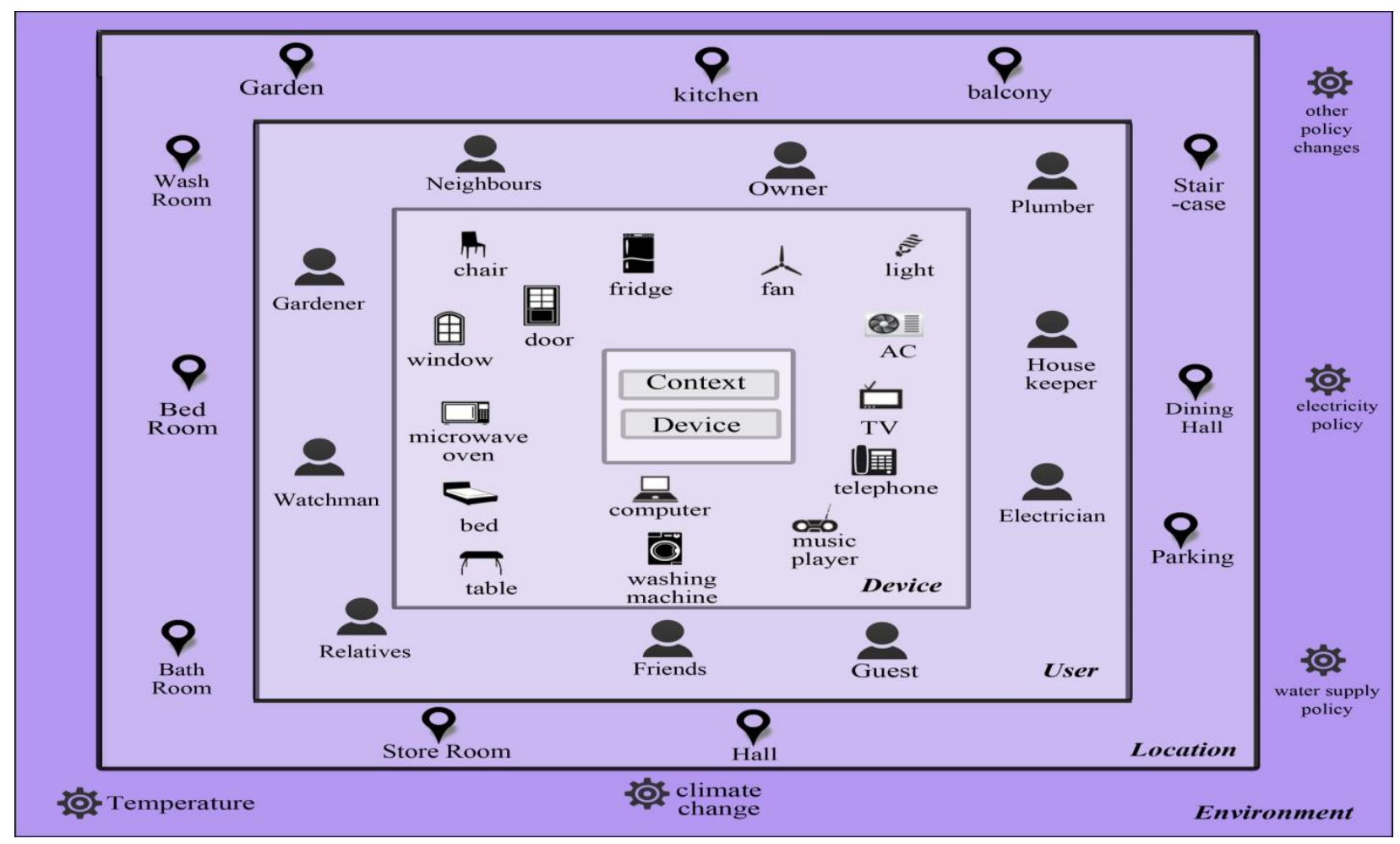

Fig 1: A Generic Middleware Model for Smart Home Environment

\section{Environment Layer}

In the generic middleware model of smart home, the environment layer depicts the change that occurs in the smart home environment. Some of the changes that are considered in the smart home environments are change in electricity policy which allows the occupant of the smart home to reduce electricity consumption, change in water supply policy that makes proper usage of resource. Some other environmental context that makes changes inside of smart home is climate and temperature changes. Any kind of environmental context can be added to the smart home environment with the help of generic middleware model.

\section{EVALUATION OF PROPOSED MODEL USING VARIOUS SMART HOME ENVIRONMENT}

The generic middleware model of smart home, which is developed to built various generic smart home environment.

Before the development of generic middleware model, smart home with specific contexts are built. But, It allows to built various kind of smart home based on the user or occupant requirements. The generic middleware model of smart home is evaluated using two different cases of smart home environments as shown below.

\section{A. Case 1: Smart Home1}

The smart home 1 environment is shown in figure 2 . The above smart home includes contexts like User context, Device context, Location context, Activity context, time context. The user layer of smart home1 has users like owner and guest. The device layer involves device like light, oven, television, air conditioner. The locations in smart home1 are hall, bedroom and kitchen.

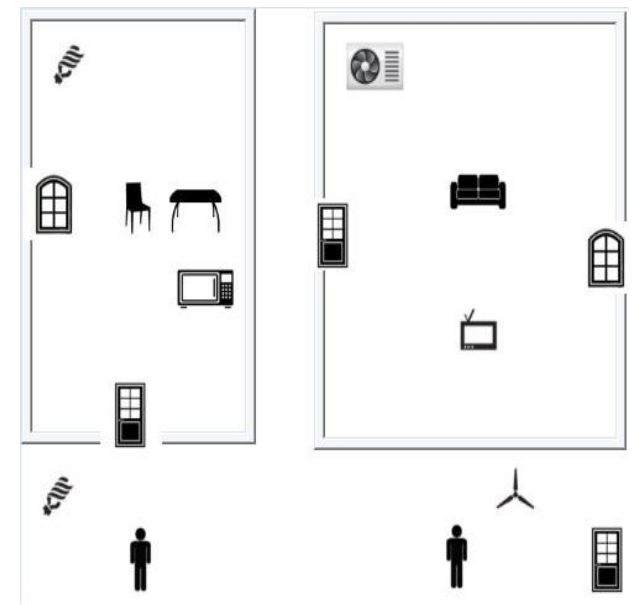

Fig 2: Representation of Smart Home 1 
The scenarios that are possible in smart home 1 are represented based on the user's perspectives. The users considered in the case of smart home 1 are user that is occupant of the home and the guest. The user of smart home 1 can access all the services that are available based on the context. The locations involved in this case are hall, room and kitchen. If user enters into the location hall, the devices like light and fan are automatically turned ON. If the user leaves, the devices will turned OFF.
The figure 3 represents the scenarios that are possible on user perception. The Guest can access the device with restrictions. If guest enters the home, notification will be sent to the Owner. The Guest can access the devices only from hall and room. The device in the kitchen can't be used. The possible actions based on guest perception are shown in figure 4.

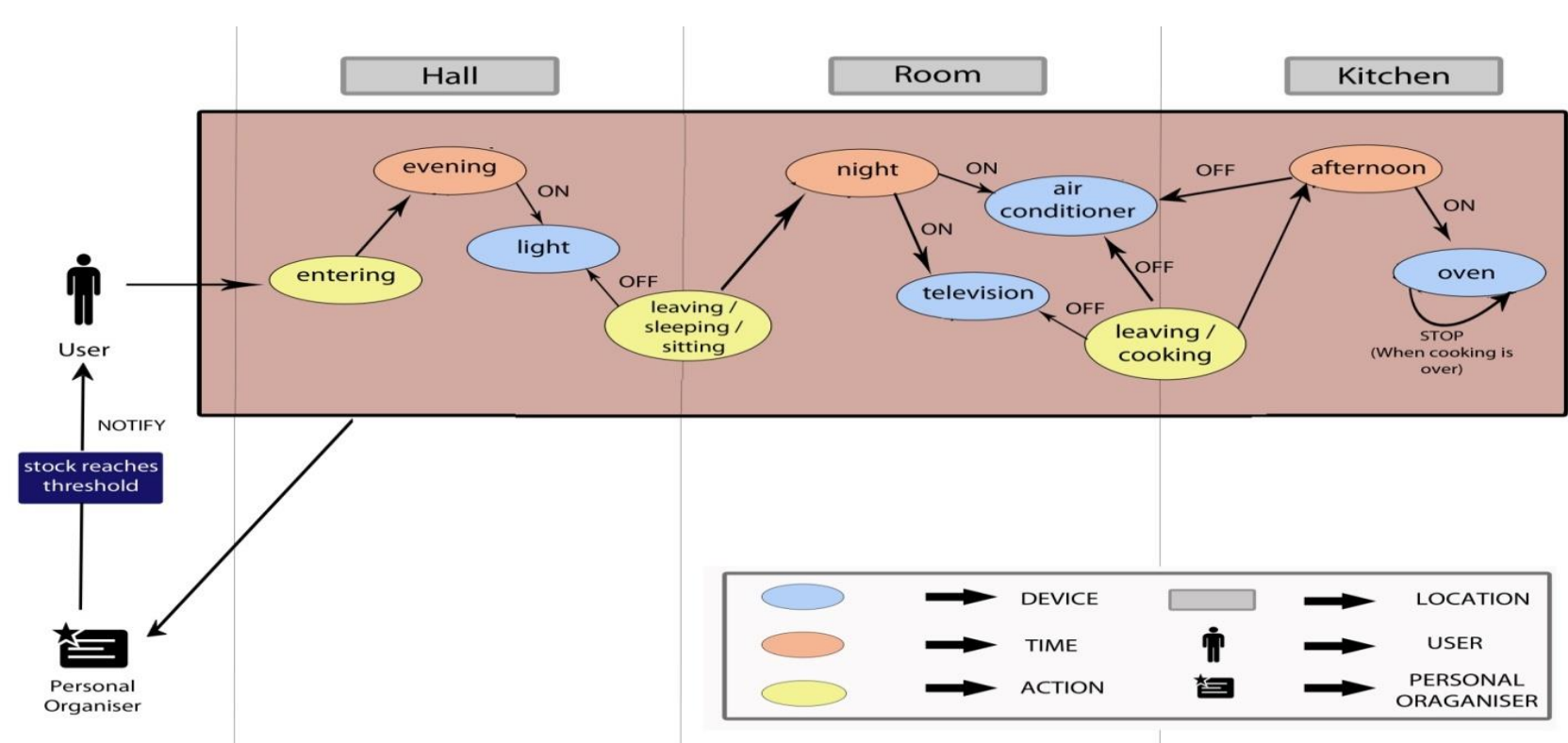

Fig 3: Scenarios of Smart Home 1 on User Perception

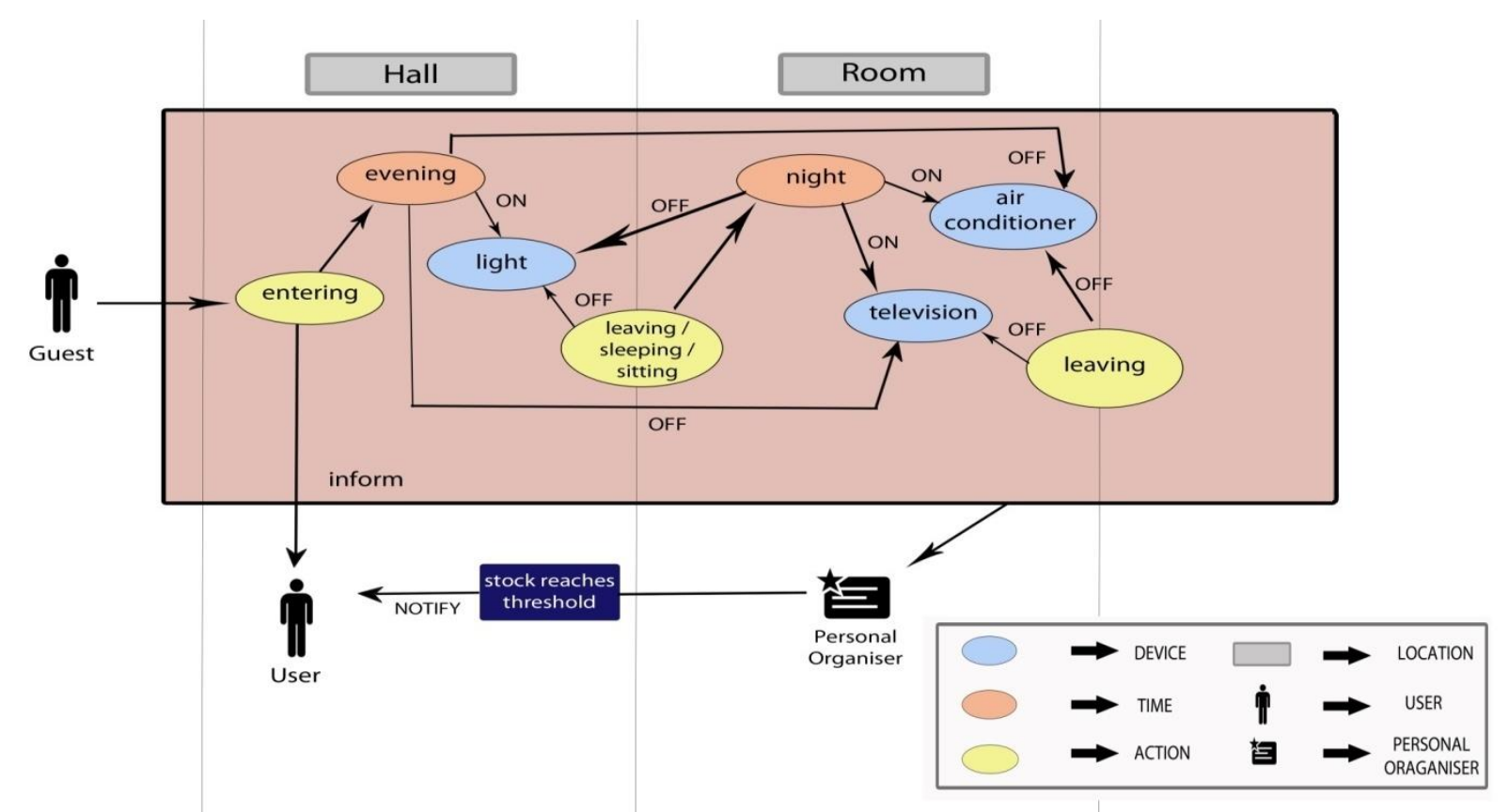

Fig 4: Scenarios of Smart Home 1 on Guest Perception

\section{B. Case 2: Smart Home 2}

The smart home 2 environment is shown in figure 5 . The above smart home includes contexts like User context, Device context, Location context, Activity context, time context. The user layer of smart home 2 has users like owner and servant. The device layer involves device like light, fan, chair, bed, sofa, telephone, oven, television, air conditioner. The locations in smart home 2 are hall, bedroom and kitchen. 


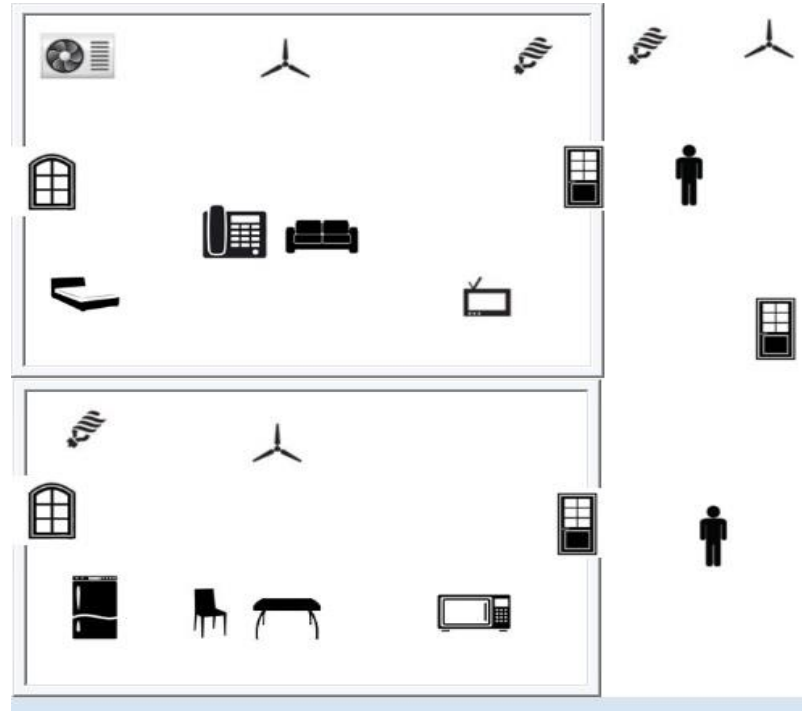

Fig 5: Representation of Smart Home 2

The Scenarios that are possible in smart home2 are represented in the following figure 6 and figure 7 based on the users perspectives. In this case, we consider two users namely owner of the home and the servant. On each perspective, the possible scenarios are represented in the following figure 6 and figure 7.

The scenarios that are possible in smart home 2 are represented based on the user's perspectives. The users considered in the case of smart home 2 are user that is owner of the home and the guest. The user of smart home 2 can access all the services that are available based on the context. The locations involved in this case are hall, room and kitchen. If user enters into the location hall, the devices like light and fan are automatically turned ON. If the user leaves, the devices will turned OFF.

The devices in the locations like room and kitchen are turned ON, when the user enters into the locations. Some other possible activities are sleeping, sitting, etc.

If user leaves from the location of smart home 2 like room and kitchen, the devices will be tuned automatically to OFF state. The figure 6 represents the scenarios that are possible on user perception.

The servant can access the devices in smart home 2 with some restrictions. If servant enters the home, notification will be sent to the Owner. The servant can access the devices that are in required location. The devices like light, fan, oven, and refrigerator can only be accessed.

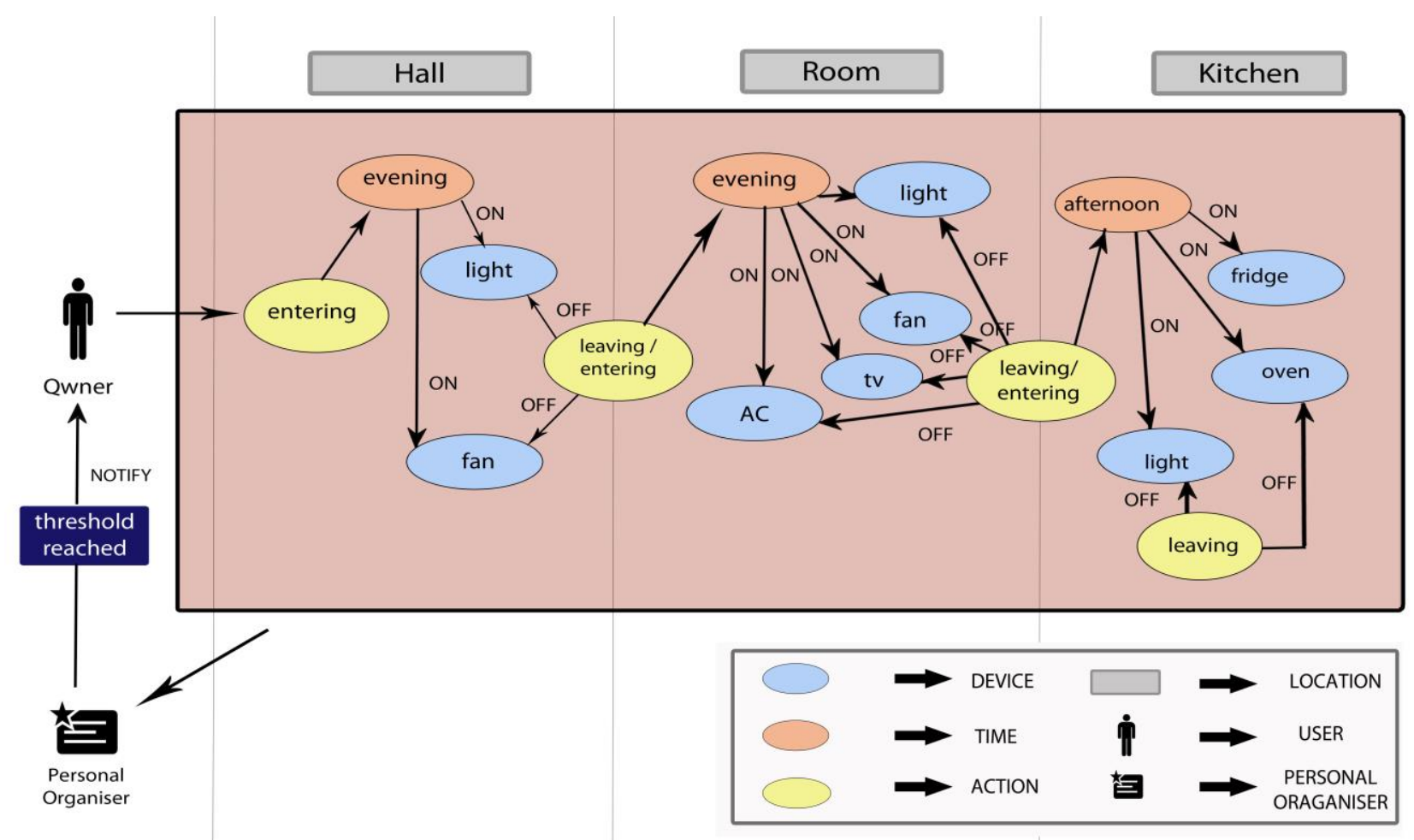

Fig 6: Scenarios of Smart Home 2 on Owner Perception 


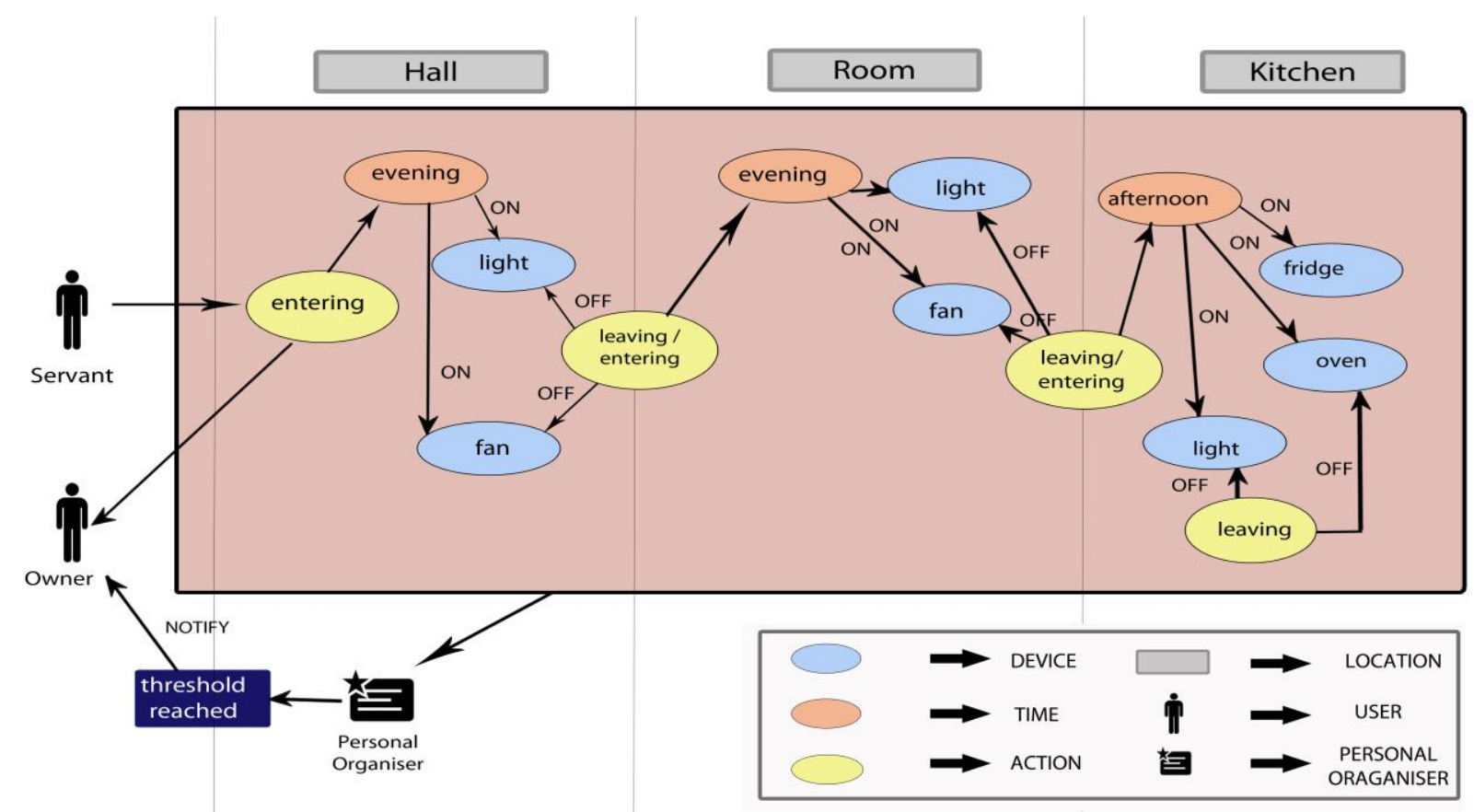

Fig 7: Scenarios of Smart Home 2 on Servant Perception

\section{CONCLUSION}

In this paper, a generic middleware model for smart home is provided. This provides the users to create or built various smart home environments of their requirements. With the generic middleware model, the service can be provided to the occupant in the smart home environment. Any number of users, locations, devices, environment changes can be added to the smart home environment. It is shown with two different cases of smart home environment with various users, devices, locations. Our future work focuses on the deployment of this model in real time.

\section{REFERENCES}

[1] Richard Harper, "Inside the Smart Home", Springer, 2003

[2] Kara Saul-Rinaldi, Robin LeBaron and Julie Caracino, "Making Sense Of The Smart Home", National Home performance council, 2013.

[3] M. Satyanarayanan, "Pervasive Computing: Vision and Challenges", To appear in IEEE Personal Communications, 2001

[4] DebashisSaha and Amitava Mukherjee, "Pervasive Computing: A Paradigm for the 21st Century", Published by the IEEE, ISSN 0018-9162, March 2003.

[5] G. Chong, L. Zhihao and Y. Yifeng, "The Research and Implement of Smart Home System Based on Internet of Things", International Conference on Electronics, Communications and Control (ICECC), pp. $2944-2947$ 2011, Sept. 2011.

[6] D. Bottazzi , A. Corradi and R. Montanari, "ContextAware Middleware Solutions for Anytime and Anywhere Emergency Assistance to Elderly People", IEEE Communications Magazine, vol.44 no.4, pp.82-90, September 2007.
[7] Diane J. Cook [IEEE Fellow], Aaron S. Crandall [IEEE Member], Brian L. Thomas [IEEE Member], and Narayanan C. Krishnan, "CASAS: A Smart Home in a Box”, Computer (Long Beach Calif), 2013.

[8] Karen Henricksen, Jadwiga Indulska, Ted McFadden, Sasitharan Balasubramaniam, "Middleware for Distributed Context-Aware Systems", Confederated international conference on On the Move to Meaningful Internet Systems, Springer, 2005.

[9] Markus Eisenhauer; Peter Rosengren; Pablo Antolin. "A development platform for integrating wireless devices and sensors into Ambient Intelligence systems." IEEE, 2009.

[10] Jeffrey C. King. "ATLAS: A Service-Oriented Sensor and Actuator Network Platform To EnableProgrammable Pervasive Computing Spaces." Thesis, 2007.

[11] Abdelsalam (Sumi) Helal, Hen-I Yang, Jeffrey King, and Raja Bose. "Atlas - Architecture for Sensor Network Based Intelligent Environments". ACM, 2007.

[12] Soma Bandyopadhyay, Munmun Sengupta, Souvik Maiti, Subhajit Dutta. "A Survey of Middleware for Internet of Things". WiMo/CoNeCo 2011, CCIS 162, pp. 288-296, 2011.

[13] Hongbo Ni, Bessam Abdulrazak, Daqing Zhang, Shu Wu. "CDTOM: A Context-driven Task-oriented Middleware for Pervasive Homecare Environment", International Journal of UbiComp (IJU), Vol.2, No.1, January 2011.

[14] Richardo Couto Antunes de Rocha, Markus Endler, "Middleware: Context Management in Heterogeneous, Evolving Ubiquitous Environments", IEEE Distributed Systems, 2006.

[15] V. Indhumathy, A. Kalaiselvi, J. Madhusudanan, V. Prasanna Venkatesan, "A Generic Simulation Tool for Pervasive Devices Application", International Journal of Engineering Research \& Technology (IJERT), 2012.

[16] A. Kalaiselvi, V. Indhumathy, J. Madhusudanan, V. Prasanna Venkatesan, "Implementation of Generic Context Middleware for Context-Aware Applications", 
International Journal of Engineering Research \& Technology (IJERT), 2012.

\section{Authors' Profiles}

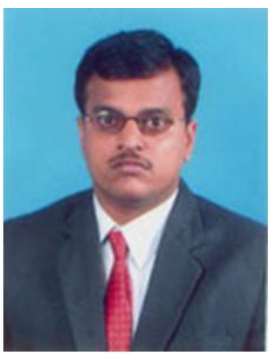

Mr. J. Madhusudanan, Associate Professor, Department of Computer Science and Engineering in Sri Manakula Vinayagar Engineering College. He holds M.E in Computer Science and Engineering and pursuing his Ph.D in Banking Technology from Pondicherry University, India. His areas of Interest are Context Aware Computing, Pervasive Middleware, Ubiquitous Computing and Smart Banking. $\mathrm{He}$ has many International and National Journal Publications.

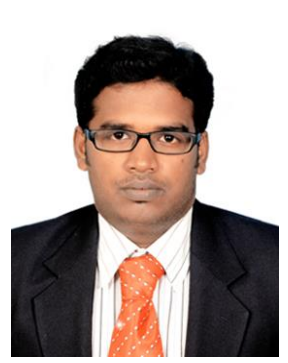

Mr. S. Hariharan. PG Scholar, Sri Manakula Vinayagar Engineering College, Puducherry. He holds B.E in Computer Science and Engineering and pursuing his M.Tech Networking in Sri Manakula Vinayagar Engineering College, Puducherry. His areas of interests are Computer Networks,
Context Aware Computing, Pervasive Middleware, and Ubiquitous Computing.

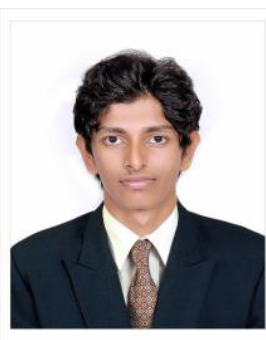

Mr. A. Manian Selvan. UG Scholar, Sri Manakula Vinayagar Engineering College, Puducherry. $\mathrm{He}$ is currently pursuing his B.Tech Computer Science and Engineering in Sri Manakula Vinayagar Engineering College, Puducherry. His area of interests is Ubiquitous Computing.

Pervasive Middleware and

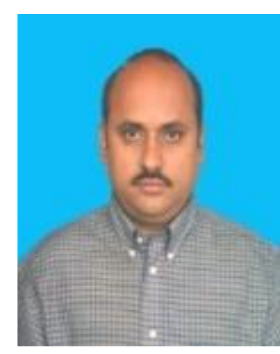

Dr. V. Prasanna Venkatesan, Associate Professor, Dept. of Banking Technology, Pondicherry University, Puducherry, India. He has more than 20 years teaching and research experience in the field of Computer Science and Engineering. His research interest includes software engineering, Business intelligence, Software Architecture and banking technology. He has designed Multilingual Compiler. $\mathrm{He}$ has many international Journal publications. He is coauthor of the book titled as Service Composition and Orchestration: Concepts and Approaches published by Vdm Verlag Dr. Müller e.K.

How to cite this paper: Madhusudanan J., Hariharan S., Manian A. Selvan, V. Prasanna Venkatesan,"A Generic Middleware Model for Smart Home", IJCNIS, vol.6, no.8, pp.19-25, 2014. DOI: 10.5815/ijcnis.2014.08.03 